\title{
Deep Learning for Classification of Compton Camera Data in the Reconstruction of Proton Beams in Cancer Treatment
}

\author{
Jonathan N. Basalyga ${ }^{1, *}$, Carlos A. Barajas ${ }^{1}$, Matthias K. Gobbert ${ }^{1}$, Paul Maggi², and Jerimy Polf ${ }^{2}$ \\ ${ }^{1}$ Department of Mathematics and Statistics, University of Maryland, Baltimore County \\ ${ }^{2}$ Department of Radiation Oncology, University of Maryland School of Medicine
}

\begin{abstract}
Real-time imaging has potential to greatly increase the effectiveness of proton beam therapy in cancer treatment. One promising method of real-time imaging is the use of a Compton camera to detect prompt gamma rays, which are emitted by the beam, in order to reconstruct their origin. However, because of limitations in the Compton camera's ability to detect prompt gammas, the data are often ambiguous, making reconstructions based on them unusable for practical purposes. Deep learning's ability to detect subtleties in data that traditional models do not use make it one possible candidate for the improvement of classification of Compton camera data. We show that a suitably designed neural network can reduce false detections and misorderings of interactions, thereby improving reconstruction quality.
\end{abstract}

Copyright line will be provided by the publisher

\section{Introduction}

The primary advantage of proton beams in cancer treatment as compared to other forms of radiation, such as $\mathrm{x}$-rays, is their finite range. The radiation delivered by the beam reaches its maximum, called the Bragg peak, at the very end of the beam's range. Little to no radiation is delivered beyond this point. By exploiting this property, it is possible to irradiate cancerous tissues, while greatly reducing the radiation delivered to, and thus damage to, the healthy surrounding tissues [1].

However, limitations exist in our ability to take full advantage of the dose delivery properties of the proton Bragg peak. These limitations are due to uncertainties in our ability to determine the Bragg peak location in the body relative to important organs that should not be irradiated. This is illustrated by Figure 1, which compares two possible beam trajectories through a cross-section of the chest [1]. The heart, outlined in purple, is positioned at top-center of the figure and a tumor, outlined in green is located next to it. The optimal trajectory, shown in the left image, uses a single beam applied in the space between the dashed white lines, to fully irradiate the tumor while stopping before reaching the heart. However, due to uncertainty in the exact location that the Bragg peak occurs (and the beam stops), represented as an orange strip at the end of the beam partially overlapping with the heart, to fully cover the tumor would mean possibly irradiating the heart. Therefore, in practice the trajectory in the right image using two beams is used. Because this trajectory passes through the lungs, delivering a small dose of radiation to them, it is considered suboptimal [1].

In order to exploit the full advantages of proton therapy, many researchers are investigating methods to image the beam in real time as it passes through the patient's body [1]. One proposed method for real time imaging is by detecting prompt gamma rays that are emitted by the beam using a Compton camera. As the proton beam enters the body, protons in the beam interact with atoms in the body, emitting prompt gamma rays. These prompt gamma rays exit the body, some of which interact with the Compton camera. Modules within the Compton camera record interactions with energy levels above some trigger-threshold. For each interaction (that is, Compton scatter), an $(x, y, z)$ location and the energy deposited are recorded. The collection of all interaction data that a camera module collects during a readout cycle is referred to as an event [2].

* Corresponding author: e-mail bajona1@umbc.edu
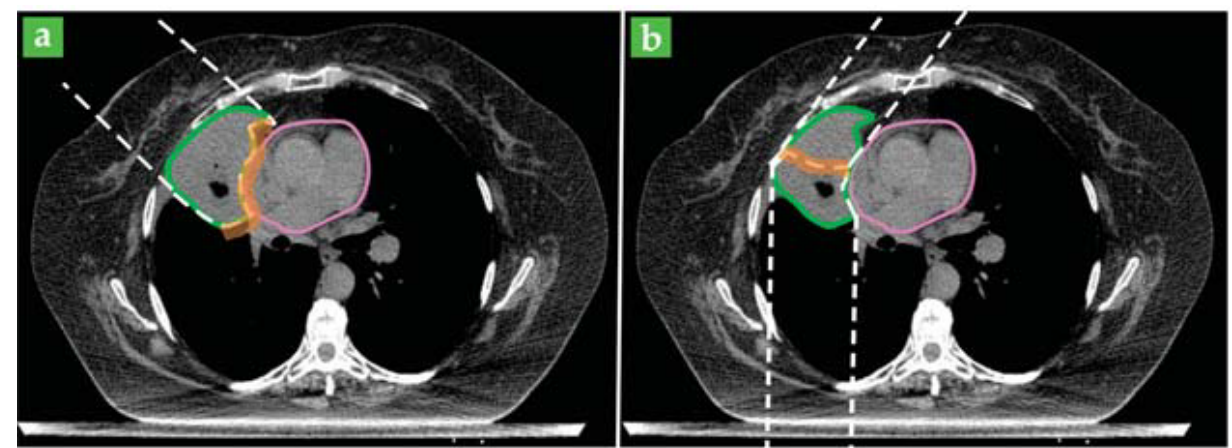

Fig. 1: (a) Optimal proton beam trajectory. (b) Suboptimal trajectory necessary to protect heart. 
Reconstruction methods require that all interactions in an event be chronologically ordered by their occurrence and that they all originate from the same prompt gamma ray. However, this is not always the case in the raw data the Compton camera outputs. Whenever these requirements are violated, that is, whenever interactions in the Compton camera data are misordered or comprised of camera interactions originating from different prompt gamma rays, the result is noise in the final reconstruction [2]. We propose that a neural network can be trained to determine if a given event is "false" or, if true, the correct camera interaction ordering. A more complete discussion is available in [3].

\section{Results: Classifying a Complete Event}

We created a fully connected neural network which we use to classify the data. The data itself is a collection of events each with three interactions. If an event only had two valid interactions we add a third interaction of all zeros to keep the event vector a consistent 15 features long. Our input layer consists of a dense 15 neuron layer. The hidden layers are 24 fully connected layers with a 4096 square dimension. Our output layer is a dense 24 neuron layer. We use a residual skip every four layers. We fix learning rate at $10^{-6}$ and batch size at 8192 , and we train for over 1000 epochs.

Figure 2 (a) shows the training and validation accuracy of this new network averaged over all five event types. For a given input, the network is trained to understand the subtle differences in interactions to determine which of the five types of events is the correct classification. These include a True Double (a gamma ray that scatters twice in the camera), a False Double (an interaction incorrectly output by the camera as a single gamma ray that scatters twice), a True Triple (a gamma ray interaction that scatters three times in the camera), a False Triple (an interaction incorrectly output by the camera as a single gamma ray that scatters three times in the camera), and Double to Triple (a True Double that was combined with another interaction and read out as a gamma ray that scattered three times in the camera). For the identification of these five types of events, the network achieves a validation accuracy of about $82 \%$.

Figure 2 (b) shows the validation accuracy for each individual event type. This shows that the network performs better on some event types than others. The network classifies Double to Triple events with an accuracy of over 90\%, False Doubles and False Triples are classified with an accuracy of slightly more than $80 \%$, while True Doubles and True Triples are correctly classified less than $80 \%$ of the time. This is possibly due to an input class invariance, that is, the frequency of occurrence differs across interaction types, so the network can incorporate more information into its model about some input classes than it can about others.

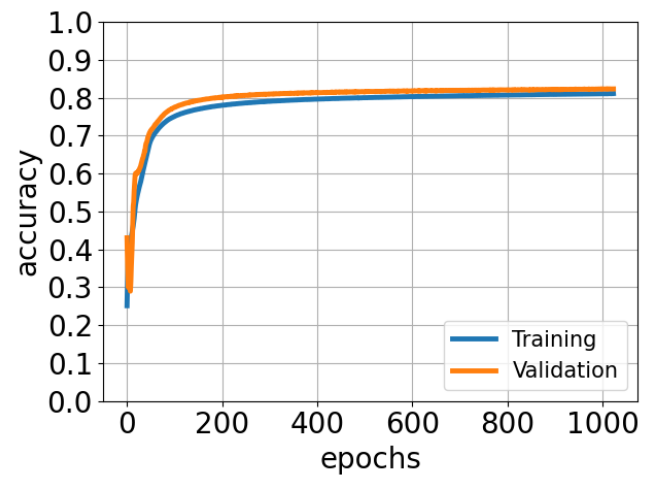

(a)

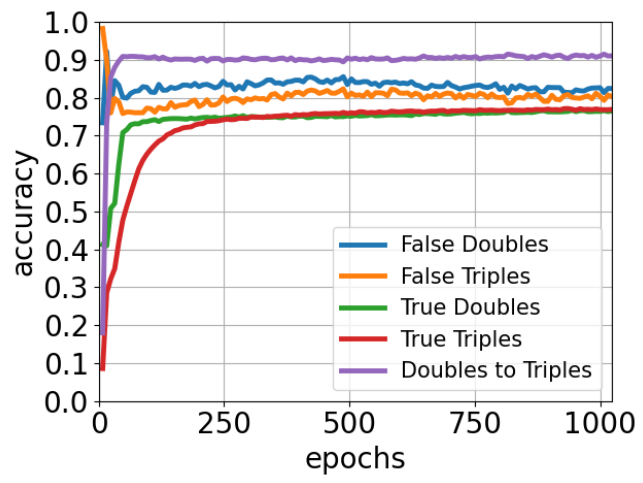

(b)

Fig. 2: (a) Training and validation accuracy averaged over all five event types. (b) Validation accuracy for each individual event type.

While the accuracy Figure 2 only reaches $82 \%$ and could likely still be improved, this is the first instance, to our knowledge, that deep learning has been applied to the classification and ordering of Compton camera interactions. Our results show that it is in fact feasible to apply a fully connected neural network to this problem. Further tests as well as discussions of the effect classification has on actual reconstructions are provided in [3].

Acknowledgements This work is supported in part by the U.S. National Science Foundation under the CyberTraining (OAC-1730250) and MRI (OAC-1726023) programs. The hardware used in the computational studies is part of the UMBC High Performance Computing Facility (HPCF). Co-author Carlos A. Barajas was supported as HPCF RA as well as as CyberTraining RA.

\section{References}

[1] J. C. Polf and K. Parodi, Phys. Today 68(10), 28-33 (2015).

[2] P. Maggi, S. W. Peterson, R. Panthi, D. S. Mackin, H. Yang, Z. He, S. Beddar, and J. Polf, Phys. Med. Biol. (online April 22, 2020).

[3] J. N. Basalyga, G. C. Kroiz, C. A. Barajas, M. K. Gobbert, P. Maggi, and J. Polf, Use of deep learning to classify Compton camera based prompt gamma imaging for proton radiotherapy, Tech. Rep. HPCF-2020-14, UMBC High Performance Computing Facility, University of Maryland, Baltimore County, 2020. http://hpcf.umbc.edu. 